\title{
A Thorough Genital Examination is the Key
}

\begin{abstract}
Keywords: Incontinence; Female hypospadias; Imperforate hymen; Genital examination

\section{Abstract}

We report a case of lifelong urine incontinence in a 9-year-old girl who underwent numerous diagnostic modalities and treatment without success. A thorough genital examination revealed a mild female hypospadias with imperforate hymen and unlocked the mystery behind her refractory state for treatment. This association led to urine accumulation within the vagina with subsequent continuous dribbling. Her condition was treated by hymenectomy.
\end{abstract}

\section{Introduction}

Urinary incontinence is commonly managed by healthy dietary habits, constipation treatment and micturition calendars. Refractory incontinence should raise the possibility of anatomic abnormalities and warrants referral to a pediatric urologist [1]. Diagnosis can be made by a simple physical exam and obviate the need for unnecessary radiological investigations.

\section{Case Report}

A 9-year-old girl was referred for daytime wetting and nocturnal enuresis. She voids 6 times per day with good quantities but continuous dribbling was noted. There was no urgency, frequency or previous surgeries. Four years ago, urodynamic studies in another institution showed no detrusor overactivity at the filling phase, a capacity of $160 \mathrm{ml}$, low detrusor pressure and no post-void residue. CT urogram revealed normal kidneys and ureters with Urine filling the vagina (Figure 1). Her pediatric urologist taught her the correct voiding posture with opening thighs without improvement. Current examination of the external genitalia revealed a normal appearing vulva, one orifice leaking urine and an imperforate hymen (Figure 2A). Under general anesthesia, hymenectomy was performed and a hypospadic urethra was noted (Figure2B-2C). Cystovaginoscopy was within normal limits. Postoperatively, patient became continent. One year later, she is still doing well.

\section{Discussion}

Urinary incontinence is a common condition in pediatrics. Establishing healthy dietary habits, treating constipation and maintaining regular voiding pattern constitute the mainstay of therapy [1]. Anatomic abnormalities are the main contributors to refractory incontinence and the need for referral to a specialist. To our knowledge, this is the first reported case of urinary incontinence in girls secondary to the association of female hypospadias with imperforate hymen. This association led to urine accumulation within the vagina with subsequent continuous dribbling [2]. History and urogenital examination are enough to establish the diagnosis for timely treatment and minimizing unnecessary radiological investigations. However, when physical exam is non-revealing, further diagnostic modalities should be done to exclude an ectopic ureter associated with a double collecting system or a congenital vesicovaginal fistula in girls with dribbling [3-5].

\section{Journal of}

Pediatrics \& Child Care

Marianne Abdo Alam ${ }^{1 *}$, Smart Najib Zeidan ${ }^{1}$, David Raja Breiche $^{2}$ and Nabil Adel Diab ${ }^{1}$

${ }^{\prime}$ Department of Pediatric Surgery, University of Balamand, Lebanon ${ }^{2}$ Department of Pediatrics, Bekaa Hospital, Lebanon

*Address for Correspondence:

Marianne Alam, University of Balamand, Faculty of Medicine \& Medical sciences, Beirut, 0096170234 782, Lebanon, E-mail: marianne.alam@gmail.com

Submission: 03 May, 2018

Accepted: 18 May, 2018

Published: 28 May, 2018

Copyright: () 2018 Alam MA, et al. This is an open access article distributed under the Creative Commons Attribution License, which permits unrestricted use, distribution, and reproduction in any medium, provided the original work is properly cited.

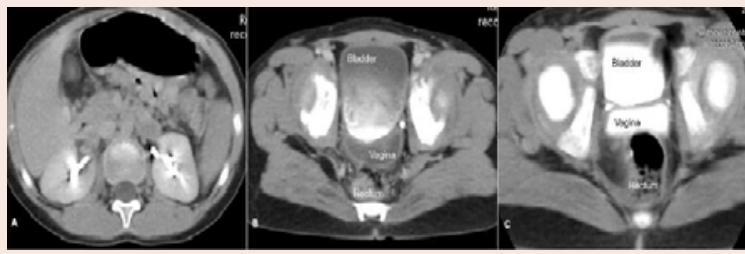

Figure 1: Axial slices of the abdomen pelvis CT scan. A) Nephrogenicexcretory phase outlining normal kidneys and normal excretory function. B) End-excretory phase with contrast filling the bladder. C) Contrast filling the vagina in delayed excretory phase.

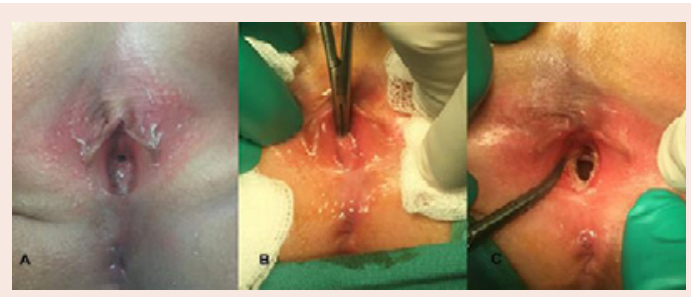

Figure 2: Physical exam and treatment. A) Normally positioned anus, inflammatory vestibule and imperforate hymen. B,C) Hymenectomy done revealing a hypospadic urethra.

\section{References}

1. Haid B, Tekgul S (2017) Primary and secondary enuresis: pathophysiology, diagnosis, and treatment. Eur Urol Focus 3: 198-206.

2. Su CC, Chang SJ, Yang SS (2014) Postvoiding urine accumulation in the vestibule in a girl complaining of postvoiding urine incontinence. Low Urin Tract Symptoms 6: 185-186.

3. Carrico C, Lebowitz RL (1998) Incontinence due to an infrasphincteric ectopic ureter: why the delay in diagnosis and what the radiologist can do about it. Pediatr Radiol 28: 942-949.

4. Jain KA (2007) Ectopic vaginal insertion of an obstructed duplicated ureter in an adult female: demonstration by magnetic resonance imaging. Clin Imaging 31: $54-56$

5. Martínez Escoriza JC, Palacios Marques AM, Lopez Fernandez JA, Feliu Rey E, Martín Medina P, et al. (2014) Congenital vesicovaginal fistula with or without menouria: a literature review. Eur J Obstet Gynecol Reprod Biol 175: $38-48$ 